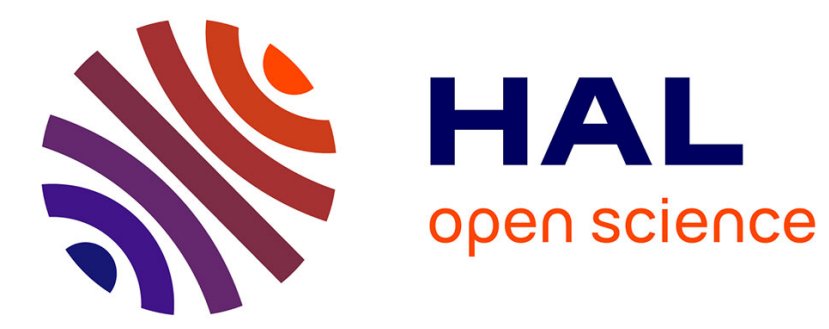

\title{
Violences conjugales, genre et criminalisation : synthèse des débats américains
}

François Bonnet

\section{To cite this version:}

François Bonnet. Violences conjugales, genre et criminalisation: synthèse des débats américains . Revue française de sociologie, 2015, 56 (2), pp.357-383. halshs-01175727

\section{HAL Id: halshs-01175727 https://shs.hal.science/halshs-01175727}

Submitted on 17 Jul 2015

HAL is a multi-disciplinary open access archive for the deposit and dissemination of scientific research documents, whether they are published or not. The documents may come from teaching and research institutions in France or abroad, or from public or private research centers.
L'archive ouverte pluridisciplinaire HAL, est destinée au dépôt et à la diffusion de documents scientifiques de niveau recherche, publiés ou non, émanant des établissements d'enseignement et de recherche français ou étrangers, des laboratoires publics ou privés.

$$
\text { Copyright }
$$




\section{VIOLENCES CONJUGALES, GENRE ET CRIMINALISATION : SYNTHÈSE DES DÉBATS AMÉRICAINS}

\section{François Bonnet}

Presses de Sciences Po (P.F.N.S.P.) | « Revue française de sociologie »

2015/2 Vol. 56 | pages 357 à 383

ISSN 0035-2969

ISBN 9782724634235

Article disponible en ligne à l'adresse :

http://www.cairn.info/revue-francaise-de-sociologie-2015-2-page-357.htm

\section{Pour citer cet article :}

François Bonnet, «Violences conjugales, genre et criminalisation : synthèse des débats américains », Revue française de sociologie 2015/2 (Vol. 56), p. 357-383.

Distribution électronique Cairn.info pour Presses de Sciences Po (P.F.N.S.P.).

(C) Presses de Sciences Po (P.F.N.S.P.). Tous droits réservés pour tous pays.

La reproduction ou représentation de cet article, notamment par photocopie, n'est autorisée que dans les limites des conditions générales d'utilisation du site ou, le cas échéant, des conditions générales de la licence souscrite par votre établissement. Toute autre reproduction ou représentation, en tout ou partie, sous quelque forme et de quelque manière que ce soit, est interdite sauf accord préalable et écrit de l'éditeur, en dehors des cas prévus par la législation en vigueur en France. Il est précisé que son stockage dans une base de données est également interdit. 


\title{
Violences conjugales, genre et criminalisation : synthèse des débats américains
}

\author{
François BONNET
}

\begin{abstract}
Résumé. Ce travail porte sur la littérature américaine sur les violences conjugales. Plusieurs grandes enquêtes ont été menées par des chercheur-e-s aux orientations théoriques différentes (violences familiales versus violence contre les femmes), et les résultats de ces enquêtes ont fait l'objet de débats. Cette revue de littérature entend restituer ces débats - sur la symétrie de genre et la bidirectionnalité des violences, sur leur définition, sur leur étiologie, sur leur traitement pénal - afin de contribuer à la construction de l'objet « violences conjugales ». La réprobation que suscitent ces violences constitue un objet d'analyse en soi.
\end{abstract}

Mots-clés. Violence conjugale - ViolenCE contre les feMmes - GenRE - CriminalisATION - ÉTATS-UNIS

Les violences conjugales sont aujourd'hui théorisées en France dans la littérature scientifique, dans la statistique publique et dans les politiques publiques comme « violences contre les femmes ». Cette approche est également dominante aux États-Unis, mais au terme d'un long débat avec l'approche dite des «violences familiales ». Ces deux approches sont fondées sur des définitions différentes de la violence, et des enquêtes différentes. Cette revue de littérature entend restituer les débats théoriques et les résultats empiriques publiés dans les revues américaines, avec des données principalement issues du monde occidental. Ces données montrent que la grande majorité des faits de violence mesurés par les enquêtes sont des disputes communes à tous les couples (hétérosexuels et homosexuels), le plus souvent dans un contexte de pauvreté et de consommation d'alcool. Les enquêtes qui mesurent les violences commises par les deux conjoints montrent une bidirectionnalité ${ }^{1}$ de ces violences. L'approche «violence contre les femmes » produit et mobilise des données qui ne permettent pas de distinguer des types de violence que la théorie féministe distingue justement. La criminalisation et la pénalisation excessives des violences conjugales suscitent des critiques d'universitaires et de militant-e-s dans le contexte racialisé de l'incarcération de masse aux États-Unis.

Les violences conjugales, et plus généralement les violences interpersonnelles au sein de la famille, forment un objet problématique. L'idée de famille comme entité

Je remercie Sébastien Chauvin, Pauline Delage, Claire Dupuy, Raul Magni-Berton et Francesco Ragazzi pour leurs utiles suggestions critiques.

1. Idée que les violences vont dans les deux sens ; elles sont mutuelles, elles ne sont pas « unidirectionnelles ». On emploie aussi ce mot pour rendre compte des violences entre supporters de football ou des rixes à la sortie des boîtes de nuit. 
rassurante et indispensable pour l'épanouissement de chacun est très largement partagée, comme norme sociale et comme savoir scientifique en psychologie et en sociologie. Or, les violences au sein de la famille sont les plus fréquentes. La violence concerne à la fois les couples, les adultes envers les enfants, les enfants entre eux et les adultes envers les personnes âgées, ce qui induit une fluidité dans les statuts d'auteur et de victime au cours de la vie (Tolan et al., 2006). La définition de ce qui est catégorisé comme violence varie dans le temps, dans l'espace et dans la stratification sociale. Les punitions des enfants sont emblématiques de cette variation (Gelles, $1985)$; la légitimité du recours à la violence dans les couples varie également. Les violences familiales surviennent dans la vie privée, laquelle a longtemps été, compte tenu des institutions légales contemporaines, largement protégée de la surveillance par les pouvoirs publics (Stinchcombe, 1963).

L'étude sociologique des violences conjugales aux États-Unis a commencé dans les années 1970, dans un contexte de contestation sociale (Gelles, 1985). La sociologie des violences familiales s'est construite en opposition à la médecine et aux « mythes médicaux » (Gelles, 1985, p. 349-350). Elle s'est d'abord appuyée sur des enquêtes dans des foyers d'accueil pour femmes battues, puis sur des enquêtes statistiques aux échantillons importants. Les grandes enquêtes ont suivi des protocoles différents, en fonction des orientations théoriques des chercheur-e-s. Il en a résulté une controverse entre «violences familiales» et «violences contre les femmes ». Cette revue de littérature entend restituer ces débats - sur la symétrie de genre et la bidirectionnalité des violences, sur leur définition, sur leur étiologie, sur leur criminalisation et leur traitement pénal - afin de contribuer à la construction de l'objet « violences conjugales ». Il ressort que les violences conjugales sont des pratiques investies de sens elles suscitent à la fois réprobation et contestation, ce qui constitue un objet d'analyse en soi.

\section{Les violences conjugales sont avant tout de la " violence situationnelle de couple "}

Les Nations unies estiment que, dans le monde, entre $20 \%$ et $50 \%$ des femmes ont un jour été victimes de violences physiques de la part de leur conjoint (United Nations Population Fund, 2000). Aux Etats-Unis, selon le National Violence Against Women Survey (Nvaws), $52 \%$ des femmes ont été victimes de violences conjugales au cours de leur vie. Chaque année aux États-Unis, environ $8 \%$ des femmes subissent des violences physiques de la part d'un conjoint, et entre $1 \%$ et $3 \%$ d'entre elles des violences physiques graves (Jordan et al., 2010, p. 608 ; Bair-Merritt et al., 2008, p. 326). Ces chiffres montrent l'ampleur du phénomène, mais posent un problème de définition très simple. La violence conjugale se résume-t-elle à la violence physique grave ?

\section{Le problème de la définition de la violence}

Le sens usuel du mot «violence » est violence physique. Mais, dans les sciences sociales, ce sens est élargi pour désigner tout acte ou situation caractérisés par l'hostilité ou l'atteinte à la dignité (Scheper-Hughes et Bourgois, 2004 ; Bourgois, 2010). Dans la sociologie des violences conjugales, le consensus est d'intégrer les violences 
psychiques et verbales à la définition de ce qu'est la violence ${ }^{2}$. La liste de questions et les résultats de l'enquête israélienne (Eisikovits et al., 2004, p. 739) donnent une idée des types de violences considérées et des ordres de grandeur: on observe un long continuum entre les blessures physiques nécessitant une hospitalisation $(0,8 \%$ des femmes interviewées rapportant un incident de ce type survenu dans les 12 derniers mois), les menaces de violence $(6,1 \%)$ et le fait de se faire crier dessus $(56 \%)$.

L'usage de la définition englobante pose plusieurs problèmes. Le premier est de diluer le sens du concept de violences conjugales en utilisant comme indicateurs des comportements qui ne sont pas couramment perçus comme de la violence. Dans l'enquête de Gregory S. Merrill et Valerie A. Wolfe (2000, p. 12-15), la liste des questions comprend «jeter des objets », «être excessivement jaloux et possessif », « critiquer constamment», " faire des scandales en public », « critiquer l'apparence physique du partenaire », «mentir », « appeler le partenaire au téléphone trop souvent », « endommager des objets qui sont la propriété du partenaire ». Dans l'enquête de Lisa Eaton et al. (2008, p. 701), «négliger votre animal domestique » fait partie des items. D'où le deuxième problème : les violences psychiques et verbales sont plus labiles. La plupart des personnes enquêtées peuvent rapporter précisément des violences ayant entraîné une hospitalisation. Au contraire, mesurer et comparer des reproches dans un couple pose un problème de recueil des données, parce que les violences psychiques sont plus sujettes à interprétation (Eisikovits et al., 2004).

Le troisième problème est celui de la cohérence d'une catégorie regroupant des faits mineurs et des faits graves. Un constat récurrent sur la criminalité est que l'écrasante majorité des faits sont mineurs, tandis que les faits graves sont rarissimes. L'écrasante majorité des vols sont des vols à la tire pour des montants inférieurs à dix euros, l'écrasante majorité des violences sont des coups qui ne nécessitent pas de soins, l'écrasante majorité des consommateurs de drogues n'ont aucun problème lié à leur dépendance, etc. (Felson, 1994). Il en va de même pour les violences conjugales : «Les définitions larges de la violence dans la famille incluent les maltraitances émotionnelles et psychologiques en plus des maltraitances physiques et sexuelles. Les définitions plus étroites se focalisent seulement sur les aspects physiques et suggèrent que l'inclusion d'autres types de maltraitance diminuerait l'importance et la sévérité que l'on associe à la violence physique. D'autres perçoivent la maltraitance psychologique comme un signe avant-coureur de violence physique et sexuelle, plutôt que comme un type de maltraitance exclusif en soi. » (Eisikovits et al., 2004, p. 730).

La plupart des couples ont des conflits qui vont occasionner le haussement de la voix, tandis que les homicides (par exemple aux États-Unis) concernent 0,0013\% des couples mariés (Bureau of Justice Statistics, 2005). Le problème des questions de définition est d'amalgamer deux phénomènes différents, et d'attribuer à l'un les propriétés qui sont en fait associées à l'autre.

2. Un problème similaire se pose avec la définition de la violence policière : les humiliations sont-elles des violences, ou la violence n'est-elle que physique ? Dans la sociologie des violences policières, la question n'est pas tranchée, et certains travaux se fondent sur une définition restrictive des violences comme occasionnant des blessures physiques, tandis que d'autres utilisent une définition plus englobante. 


\section{La symétrie de genre}

Un problème plus complexe est que le recours à une définition englobante des violences dans des grandes enquêtes quantitatives produit des données où les femmes sont aussi violentes que les hommes. Ce résultat est appelé «symétrie de genre » (gender symmetry).

Cette question est centrale pour comprendre comment se structure le débat scientifique sur les violences conjugales. Plus on élargit la définition de la violence pour inclure le harcèlement et les violences psychologiques, plus la part des femmes violentes augmente, pour atteindre la majorité des faits les moins graves - c'est-à-dire les plus nombreux. Avec une définition large, les violences familiales sont autant le fait des hommes que des femmes (Fiebert, 1997 ; Archer, 2000 ; Graham-Kevan et Archer, 2003 ; Tolan et al., 2006). Ce constat est établi dès la fin des années 1970. Les enquêtes enregistrent entre 3,8 et 4 incidents par an pour 100 individus, avec les hommes légèrement plus victimes que les femmes (Gelles, 1985, p. 356). Le National Family Violence Survey de 1985 compte, pour 1000 couples, 78 incidents des violences mineures commis par des femmes, et 72 par des hommes; pour les violences graves, 46 par les femmes et 50 par les hommes (Tolan et al., 2006, p. 564). Murray A. Straus et al. (1980) découvrent que la violence est majoritairement réciproque, d'où l'idée d'étudier des conflits plutôt que des victimes.

Selon M. A. Straus, il y a plus de 150 enquêtes dont le résultat est la symétrie de genre (2006, p. 1086). La méta-analyse de 522 sources de John Archer (2000, p. 651) montre que les femmes sont plus souvent violentes que les hommes, mais que $62 \%$ des personnes blessées par un conjoint sont des femmes : "Cela a conduit un autre chercheur de conclure que les maris et les femmes sont "agressifs", mais que beaucoup plus de maris sont "violents". » (Tolan et al., 2006, p. 564). Si les enquêtes trouvent une telle "symétrie de genre », c'est que les violences sont souvent bidirectionnelles : au sein du même couple, les deux conjoints sont mutuellement violents. Plus de la moitié des violences serait bidirectionnelle, les deux quarts restants se partageant également entre violences des hommes vers les femmes, et violences des femmes vers les hommes (Straus, 2006 ; Langhinrichsen-Rohling, 2010 ; Tolan et al., 2006). Les taux d'initiative des violences (celui ou celle qui initie l'échange des coups) sont les mêmes pour les hommes et les femmes (Tolan et al., 2006 ; Langhinrichsen-Rohling, 2010).

Avec une définition restrictive de la violence (comme violence grave), le résultat de la symétrie de genre est moins marqué. Les homicides sont commis à plus de $70 \%$ par les hommes (Saunder, 2002 ; Saunders and Browne, 2000). Dans l'enquête National Violence against Women Survey, les femmes ont trois fois plus de risques d'être hospitalisées que les hommes (pour des faits de violences conjugales) et elles sont $1,1 \%$ à avoir reçu des coups lors des 12 derniers mois, contre $0,6 \%$ pour les hommes (Tjaden et Thoennes, 2000). La quasi-totalité des viols conjugaux sont commis par les hommes, ainsi qu'une majorité des violences au moment de la séparation, et le stalking ${ }^{3}$ (DeKeseredy et Dragiewicz, 2007).

La symétrie de genre ne signifie pas l'égalité dans la victimation: «Ce que la "symétrie" signifie est qu'à peu près le même nombre d'hommes et de femmes

3. Le stalking est la pratique qui consiste à suivre, traquer et harceler de manière obsessionnelle une personne contre sa volonté. Notons que dans une étude citée par J. Langhinrichsen-Rohling (2010, p. 183), $25 \%$ des femmes battues en foyer admettaient «stalker» leur conjoint violent. 
reconnaissent avoir commis au moins un acte de violence dans la liste d'un quelconque questionnaire [...]. Il est cependant clair que même dans ces travaux soi-disant "symétriques de genre" sur grands échantillons, la violence des hommes produit plus de blessures physiques, plus de conséquences psychologiques négatives et plus de peur. » (Johnson, 2010a, p. 213).

Pour le dire autrement : «Bien que les femmes agressent leur conjoint dans les mêmes proportions que les hommes, elles sont les principales victimes à cause des blessures physiques, financières et émotionnelles plus graves. » (Straus, cité dans Kimmel, 2002, p. 1348 ; corroboré par Langhinrichsen-Rohling, 2010, p. 181-182). Il n'est pas anodin qu'Evan Stark, auteur qui se définit comme féministe radical, écrive : "Je suis d'accord avec l'affirmation empirique générale de LanghinrichsenRohling, à savoir que, dans les couples, les femmes comme les hommes recourent à la violence fréquemment, que leurs motivations sont souvent similaires, et que les deux parties sont souvent violentes. » (Stark, 2010, p. 202). Correctement nuancé, le résultat empirique de la symétrie de genre est largement consensuel.

\section{Débats méthodologiques}

Le résultat de la symétrie de genre a suscité deux débats méthodologiques ${ }^{4}$ concernant l'échantillonnage (sampling). Un premier débat concerne l'opposition entre les grandes enquêtes quantitatives par sondage et les enquêtes dans les foyers pour femmes battues. Les études qui montrent que la violence est le fait des hommes proviennent de données recueillies dans les foyers pour femmes battues, où les violences sont les plus graves. Par exemple, dans la deuxième moitié de l'année 1977, les Crisis Centers des hôpitaux publics de la ville de New York ont conseillé 490 femmes battues pour deux hommes (Johnson, 1995, p. 285). Pour les chercheur-e-s qui travaillent dans les foyers et les hôpitaux, les grandes enquêtes statistiques sont aberrantes au regard de la réalité de qui est violent et de qui est blessée 5 .

Pour les tenants des grandes enquêtes quantitatives, les enquêtes dans les foyers d'accueil sont fondées sur de trop petits échantillons. Les enquêtes dans la population générale (sur des gros échantillons représentatifs de population) enregistrent tout ce que les enquêté-e-s sont prêt-e-s à déclarer. Elles permettent de recueillir des données sur les conflits mineurs (Johnson, 1995, 2006 ; Graham-Kevan et Archer, 2003), lesquelles se prêtent particulièrement au résultat de gender symmetry. Les auteurs qui s'appuient sur ces enquêtes considèrent que le résultat de la gender symmetry montre la supériorité de l'analyse statistique, qui révèle une vérité objective, débarrassée des prénotions (Tolan et al., 2006, p. 563).

Un deuxième débat, postérieur au premier, oppose deux enquêtes quantitatives. La première est celle utilisée par les chercheurs dans la tradition de la family violence autour de Murray Straus, Conflict Tactics Scale (CTS, Échelle des tactiques de conflit).

4. Un troisième problème méthodologique concerne la probabilité différentielle que les hommes et les femmes déclarent les violences. On pourrait penser que les hommes seraient portés à sous-déclarer les violences dont ils sont victimes (par honte ou par conformité aux normes de genre), ou que les femmes seraient portées à les surestimer (notamment pour les questions de divorce et de garde d'enfants). Selon
M. S. Kimmel (2002, p. 1344-1345), ces deux prénotions sont sans fondement empirique.

5. Les chercheur-e-s dans cette tradition citent souvent l'article de S. K. Steinmetz (1977) sur le «syndrome du mari battu» (battered husband syndrome) comme exemple d'artefact statistique qui ne renvoie à rien de concret dans la réalité, puisqu'aucun homme battu ne porte plainte. 
La seconde est l'enquête féministe, Violence Against Women Survey (VAWS, Enquête sur les violences contre les femmes). Pour simplifier, les enquêtes $C T S$ «tendent à trouver des taux élevés de violence conjugale, des niveaux stables de sévérité, des taux bas de blessures et trouvent ces dernières commises également par les hommes et les femmes » (Kimmel 2002, p. 1340). Les enquêtes CTS interrogent les hommes et les femmes; elles procèdent par enquête de victimation («Avez-vous subi...? ») et d'autorévélation («Avez-vous commis... ? »). L'enquête CTS a été formalisée dans un premier temps par M. A. Straus (1979). Pour répondre aux critiques, M. A. Straus et ses collègues ont formalisé une enquête CTS modifiée, CTS2, qui prend mieux en compte les blessures et les violences sexuelles au sein du couple (Straus et al., 1996). Les enquêtes CTS/CTS2 forment le gros des études et méta-analyses qui montrent la symétrie de genre comme Martin S. Fiebert (1997) et J. Archer (2000). Elles cherchent à mesurer les conflits dans les familles, ce qui est une question de recherche différente de la question des violences contre les femmes. Le paragraphe introductif du questionnaire de CTS2 qui est lu aux enquêtés énonce : "Même dans les couples qui s'entendent bien, il arrive qu'il y ait des désaccords, que l'autre personne agace, que l'on souhaite des choses différentes de l'autre, ou qu'on a tout simplement des accrochages ou des disputes parce qu'on est de mauvaise humeur, fatigué, ou pour toute autre raison. Les couples ont aussi de nombreuses façons différentes d'essayer de résoudre leurs différends. Voici une liste de choses qui peuvent arriver quand vous avez des différends. » (Straus et al., 1996, p. 310).

CTS et CTS 2 encourent plusieurs critiques : le questionnaire ne prend pas en compte les homicides et distingue mal les violences très graves des violences moins graves ; il ne s'adresse qu'aux personnes en couple, alors que les couples séparés et divorcés connaissent plus de violences, et plus souvent masculine ; et il laisse de côté la question des motivations de la violence (Saunders, 2002 ; Kimmel, 2002 ; Eisikovits et al., 2004).

Au contraire, les enquêtes VAWS « trouvent typiquement que la violence conjugale est rare, grave, s'aggrave avec le temps, et est commise en premier par les hommes » (Kimmel, 2002, p. 1338). Par exemple, les enquêtes CTS de M. A. Straus et Richard J. Gelles trouvent entre $11 \%$ et $12 \%$ de violences annuelles (pour 100 couples), tandis que les enquête VAWS de Patricia Tjaden et Nancy Thoennes (2000) ou l'enquête canadienne (citée dans Johnson, 1996) trouvent entre 1,5\% et $3 \%$ de violence annuelle. Ces disparités suggèrent d'importantes différences dans les définitions et dans le recueil des données. Le nom même de l'enquête VAWS (violences contre les femmes) indique son propos. Alors que CTS cherche à produire des données sur des conflits intrafamiliaux, VAWS est conçue pour produire des données sur les violences contre les femmes : les enquêtes VAWS n'interrogent pas les hommes, et ne demandent pas aux femmes d'autorévéler leur violence ${ }^{6}$. Les critiques qui leur sont faites sont résumées ainsi par les auteurs de l'enquête israélienne : "VAWS est conçue et mise en œuvre de telle manière qu'elle considère a priori la femme comme une victime indépendamment des circonstances. Ce biais est rendu manifeste par le fait que l'enquête ne concerne que les femmes, qu'elle exclue et ignore la contribution possible des femmes au développement d'un événement violent, et ignore les disparités possibles entre les comptes rendus des hommes et des femmes d'un même événement violent. » (Eisikovits et al., 2004, p. 732).

6. Toutes les enquêtes VAWS n'interrogent pas que les femmes : P. Tjaden et N. Thoennes (2000), par exemple, ont interrogé 8000 hommes et 8000 femmes.

362, Revue française de sociologie, 56-2, 2015 
Certaines enquêtes sont conçues pour combiner les enquêtes CTS et VAWS : les enquêtes israéliennes (Eisikovits et al., 2004) et espagnoles (Medina-Ariza et Barberet, 2003). Les financements publics majeurs vont désormais de manière préférentielle aux enquêtes VAWS aux États-Unis, au Canada (Straus, 2006) ou en France.

Il existe, enfin, un troisième type d'enquête aux États-Unis : le National Crime Victimization Survey (NCVS) et le sondage de victimation du Bureau of Justice Statistics, qui dépend du ministère américain de la justice. La comparaison, par Ronet Bachman (2000), des données issues du NCVS et de l'enquête VAWS montre que les taux de violences contre les femmes sont assez similaires, mais l'on constate une disparité importante pour le viol, mieux mesuré par VAWS $(0,26 \%$ pour le NCVS, $0,87 \%$ pour $V A W S)$.

\section{La distinction importante : le " terrorisme intime " et la " violence situationnelle de couple "}

Pour résoudre le problème de la symétrie de genre, le sociologue féministe Michael P. Johnson (1995, 2006 ; Johnson et Ferraro, 2000) a proposé une typologie des violences conjugales.

Cette typologie oppose initialement le «terrorisme patriarcal» (patriarchal terrorism) et la «violence commune de couple» (common couple violence) (Johnson, 1995). Les désignations évoluent vers «terrorisme intime» (intimate terrorism) et « violence situationnelle de couple » (situational couple violence) (Johnson et Ferraro, $2000)^{7}$. L'idée est la même. Il y a, d'une part, la violence purement sexiste, les hommes qui terrorisent leur femme, qui considèrent que le mariage est un titre de propriété. Cette violence a pour finalité le contrôle de la conjointe : contrôle des fréquentations, des dépenses, des tâches ménagères, de la sexualité. Elle ressort du « terrorisme intime », elle est masculine (à 97 \% selon M. P. Johnson, 2006, p. 1010) et aboutit aux violences et traumatismes graves dont on recueille les conséquences dans les foyers, les hôpitaux ou dans les morgues.

Il y a, d'autre part, la masse de tous les différends qui opposent à peu près tous les couples dans la vie quotidienne, qui généralement ne vont pas plus loin qu'une dispute «normale », mais qui parfois - exacerbés par les difficultés matérielles et l'alcoolisme - dégénèrent en cris, vaisselle cassée, gifles, et, très rarement, en coups, voire en coups mortels. À peu près tout le monde se dispute, hommes et femmes à parts égales, sans que cela soit lié à une volonté patriarcale de contrôle. Pour M. P. Johnson, c'est cette violence que les enquêtes CTS enregistrent (trop) bien, et qui laisse croire à la symétrie de genre.

Cette distinction pose à la fois un problème méthodologique et un problème théorique. Le problème méthodologique concerne l'identification des terroristes intimes dans la masse des couples violents. Si les enquêtes quantitatives mesurent des actes violents, comment distinguer la violence patriarcale de la violence qui résulte de différends ? Pour M. A. Straus, le concepteur des enquêtes CTS, une enquête de

7. M. P. Johnson a rajouté deux autres types à sa typologie, la « résistance violente » (principalement le fait des femmes en réaction au terrorisme intime) et le "contrôle mutuel violent », c'està-dire la présence dans le couple de deux «terroristes »(Johnson et Ferraro, 2000 ; Leone et al., 2004 ; Johnson, 2010b). Ces deux types sont d'importance secondaire. 
victimation ou d'autorévélation mesure des incidents, pas des motifs. Dans la tradition positiviste, les motifs de l'action sont inaccessibles. Des méthodes qualitatives pourraient révéler les motifs, mais ne peuvent pas faire des estimations d'ampleur relative. M. P. Johnson (1995, p. 291) le concède lui-même : "Puisque le cœur de la distinction entre violence commune de couple et terrorisme patriarcal est une différence de motif, les preuves avancées plus haut ne peuvent qu'être conjecturales. »

Le problème théorique est le suivant : le terrorisme intime et la violence situationnelle de couple sont-ils des catégories d'actes et d'individus qui existent dans la réalité, ou des idéaux-types qui n'existent que conceptuellement, pour aider les chercheurs à faire sens du réel ? La lecture des différents travaux de M. P. Johnson laisse entendre que ses catégories renvoient à des réalités concrètes : il y a d'un côté les couples qui se disputent, et, de l'autre les terroristes intimes qui ressortent de la violence patriarcale. Par exemple, en ré-analysant d'autres enquêtes, M. P. Johnson (2005) estime qu'entre $11 \%$ et $33 \%$ de toute la violence conjugale est le fait de terroristes intimes. Si l'on pose qu'il est possible de distinguer les types de violences, et que ces types de violences sont séparables en entités distinctes, la logique statistique veut que la violence situationnelle soit archi-prédominante, puisque le constat de la symétrie de genre ressort des enquêtes sur échantillons aléatoires massifs. Pour le dire autrement : si le terrorisme intime est une activité masculine, et que les enquêtes sur gros échantillons trouvent autant de femmes que d'hommes violents, c'est que le terrorisme intime est statistiquement noyé dans la masse de toutes les disputes de couples. C'est le sens d'une étude CTS 2 sur 828 jeunes adultes (dont 437 femmes et 391 hommes) en Nouvelle-Zélande. David M. Fergusson et al. (2005) rajoutent des questions afin de détecter les terroristes intimes et incluent des données médicales pour repérer les violences graves. Ce protocole, conçu pour déceler les terroristes intimes, rejoint le résultat de la symétrie de genre. En fait, pour trouver du terrorisme intime, il faut revenir à des (petits) échantillons recrutés dans des foyers de femmes battues (Johnson, 2005).

Encore une fois, cette logique de décompte repose sur une lecture dichotomique de la distinction entre violence situationnelle de couple et terrorisme intime. Cette lecture a le mérite de la simplicité, mais réifie des situations que l'on devine plus ambigües. Une approche en termes d'idéaux-types est plus sociologique, mais prive la recherche de la possibilité de compter les terroristes intimes - ou de les certifier comme tels.

\section{Deux conceptions des violences conjugales}

Les résultats constatant la symétrie de genre tendent à penser les violences conjugales comme des relations violentes plutôt que des individus violents (Fergusson et $a l .$, 2005). Le problème n'est pas de savoir qui a commencé, qui se défend, qui est légitime à être violent, mais de constater que «la plupart de la violence dans les couples est similaire en termes de fréquence, de gravité et d'initiative, et qu'elle peut être mieux expliquée du point de vue de la relation plutôt que de celui de l'imposition d'un pouvoir d'une personne sur une autre» (Tolan et al., 2006, p. 561). En clair : la violence conjugale n'est pas un problème de violences faites aux femmes, mais un problème de violence au sein de la famille (Fergusson et al., 2005). La notion de family violence présente l'avantage d'être fondée sur des relations empiriques entre violences dans le couple et violences sur les enfants. Le fait d'avoir été victime de violences pendant l'enfance maximise la probabilité d'être victime de violences dans

364, Revue française de sociologie, 56-2, 2015 
le couple (Tolan et al., 2006), et augmente la probabilité que la victime reste dans une relation violente (Gelles, 1985). Une méta-analyse de trente études montre une cooccurrence médiane de $41 \%$ de maltraitance d'enfants et de violence conjugale (cité dans Tolan et al., 2006). Les femmes ont d'ailleurs une probabilité quatre fois plus élevée que les hommes de maltraiter leurs enfants (ibid.).

Cette perspective de la family violence s'oppose à celle de la « violence contre les femmes » (violence against women). L'idée de violences contre les femmes est ancrée dans deux fondements empiriques : la gravité des violences dont les femmes sont victimes et le contexte général des inégalités de genre. Dans la conception « violences contre les femmes », les violences conjugales ne sont pas pensées comme faisant partie des violences intrafamiliales, mais de toutes les violences des hommes contre les femmes, comme les viols et le harcèlement sexuel. La cohérence conceptuelle de l'idée de violences contre les femmes repose sur l'hypothèse que toutes ces violences différentes ont pour cause la domination masculine. Dans le domaine spécifique des violences conjugales, l'une des premières formulations est la suivante : «En fait, les hommes qui agressent leur femme se conforment aux prescriptions culturelles qui sont vénérées dans les sociétés occidentales : l'agressivité, la domination masculine et la subordination de la femme - et ils utilisent la force physique comme moyen de mettre en œuvre cette domination. » (Dobash and Dobash, 1979, p. 24).

Violences et genre sont liés. Dans l'enquête ukrainienne sur les violences conjugales, le fait pour un homme de chercher à contrôler la vie de son épouse est corrélé à la violence (Barrett et al., 2012). Dans les enquêtes allemandes, finlandaises, suédoises, espagnoles et britanniques, les attitudes traditionnelles envers les femmes et les enfants sont corrélées à la violence (Kury et al., 2004). Dans le monde, plus le statut de la femme est subalterne, plus il y a de violences (Langhinrichsen-Rohling, 2010).

L'une des sources empiriques de cette conception des violences est le Duluth Model (Bumiller, 2010). Le Duluth Model est fondé sur le travail de militants associatifs basés dans le Minnesota à la fin des années 1970. Il s'appuie sur l'expérience de la souffrance des femmes accueillies dans les foyers et cherche, par une thérapie comportementale cognitive, à faire changer les hommes violents. Le Duluth Model fait l'hypothèse que la violence conjugale résulte du comportement rationnel des hommes qui visent à faire perdurer le patriarcat (Gondolf, 2007). Une étude, le Minneapolis Domestic Violence Experiment (MDVE), a montré que seule la menace de l'arrestation par la police était en mesure de dissuader les maris violents : le traitement pénal doit «renverser la socialisation masculine et éduquer les hommes » (Bumiller, 2010, p. 178).

Parler de violences familiales ou de violences contre les femmes engage donc des postures théoriques différentes. Initialement, le vocabulaire tournait autour de la notion d'épouse : wife battering, wife assault, wife abuse afin de signifier qui est la victime (Gelles, 1985). La découverte de la symétrie de genre dans les enquêtes quantitatives a fait évoluer le langage vers les idées de spousal abuse, mutual partner violence, domestic violence. Mais, selon P. Tjaden et N. Thoennes (2000, p. 143), " reformuler le problème des femmes battues [wife abuse] en un problème de violence dans le couple [spousal assault] ou de violences familiales [family violence] banalise la situation difficile des femmes battues et détourne l'attention des causes profondes de la violence [wife abuse], à savoir l'inégalité de genre et la domination masculine ».

Aujourd'hui trois concepts coexistent. Le premier est celui de intimate partner violence $(I P V)$, qui renvoie à la violence dans le couple sans préjugé d'orientation sexuelle 
ni de statut conjugal (McClennen, 2005). Le deuxième est celui de family violence, qui englobe $I P V$ et les autres types de violences et de maltraitances intrafamiliales, comprenant notamment les enfants et les personnes âgées. Le troisième concept est celui de violence against women, violences contre les femmes. L'univers théorique de la violence contre les femmes est celui de la domination de genre, à la fois dans le couple, dans l'espace public et au travail. La conséquence méthodologique d'une conceptualisation en termes de "violences contre les femmes » est de cesser d'interroger les hommes dans les enquêtes quantitatives, et de ne poser aux femmes que des questions de victimation. Ce choix a pour conséquence de faire disparaître la symétrie de genre par non-recueil des données. Comme l'écrit J. Langhinrichsen-Rohling (2010, p. 183), «cette absence de mesure de la perpétration des femmes est une stratégie d'évitement qui semble de nature à entraver plutôt qu'à faire progresser le champ ».

\section{Des causes communes dans les couples hétéro- et homosexuels}

Dans cette section, on liste les causes sociales ${ }^{8}$ des violences conjugales et l'on constate que ces causes sont similaires dans les couples hétéro- et homosexuels. La présence de violences dans les couples gays et lesbiens montre l'importance quantitative de la violence situationnelle de couple dans le volume total des violences au sein du couple.

\section{Les déterminants sociaux des violences}

La violence conjugale est corrélée à la pauvreté. Ce résultat est récurrent dans les enquêtes américaines (Gelles, 1985 ; Benson et al., 2003 ; Benson et Fox, 2004 ; Bachman, 2000 ; Tolan et al., 2006 ; Rennison et Planty, 2003 ; Bair-Merritt et al., 2008), espagnoles, allemandes, suédoises, finlandaises et britanniques (Kury et al., 2004), israéliennes (Eisikovits et al., 2004), palestiniennes (Haj-Yahia, 2000) et kényanes (Kimuna et Djamba, 2008). Dans l'enquête de Dallan F. Flake (2005), au Pérou la relation n'est pas liée à la pauvreté mais au faible niveau d'éducation. Rachel Jewkes (2002) trouve un lien quasi universel entre pauvreté et violences conjugales. Aux États-Unis, plus de la moitié des femmes qui reçoivent des aides publiques ont été victimes de violences conjugales (Sokoloff et Dupont, 2005). L'enquête israélienne fournit un bon exemple de combinaison des facteurs sociaux dans l'étiologie des violences conjugales ( $\mathrm{N}=2092$ femmes et 452 couples) (Eisikovits et al., 2004). L'homme violent est jeune, pauvre, avec un faible niveau d'éducation, marié depuis peu, musulman ou bien juif orthodoxe; la variable immigration (qui concerne par exemple les immigrés russes, éthiopiens et américains) ne joue pas.

8. Les violences conjugales intéressent à la fois les sociologues, les psychologues et les médecins. Pour les psychologues, les types de personnalité sont des facteurs explicatifs : par exemple, les personnalités, « impulsives », « antisociales » et «psychopathes » seraient 13 fois plus violentes que les autres (Tolan et al., 2006, p. 567). Parmi les prédicteurs non strictement sociaux des violences conjugales, il y a le fait d'avoir été auteur ou victime de violences, la faible estime de soi (Tolan et al., 2006, p. 567 ; Gelles, 1985, p. 358) ; la séparation récente (Jordan, Campbell and Follingstad, 2010 ; Bachman, 2000 ; Michalski, 2004); le fait que l'usage de la violence soit normal et habituel dans une société donnée (Jewkes, 2012). 
La question du lien entre pauvreté et violences conjugales a été approfondie par Michael L. Benson et Greer L. Fox (2004) et M. L. Benson et al. (2003), qui trouvent un niveau de violences graves 2,4 fois plus élevé dans les quartiers pauvres que dans les autres. Les hommes qui ont été deux fois et plus au chômage au cours de la période test ont une probabilité 2,6 fois supérieure d'être violents (Benson et Fox, 2004). Les couples qui perçoivent leur situation économique avec pessimisme ont une probabilité 3,5 fois supérieure d'être violents. Le facteur causal est-il la pauvreté en elle-même, ou la qualité du quartier ? Il semble qu'un effet de quartier joue dans la causalité des violences (Benson et al., 2003).

Callie Rennison et Mike Planty (2003, p. 438) trouvent des résultats comparables : selon les données du NCVS (l'enquête de victimation générale, plus de 650000 enquêtés), les taux de violences conjugales sont les plus élevés (2\%) dans les ménages qui gagnent moins de 7500 dollars annuels. Les femmes qui vivent dans des ménages gagnant plus de 50000 dollars annuels connaissent des taux de victimation de $0,36 \%$. La relation entre pauvreté et violence reste la même quand on considère d'autres types de violences intrafamiliales comme les violences contre les enfants ou la maltraitance des personnes âgées (Tolan et al., 2006 ; Kury et al., 2004). Selon R. Jewkes (2002) et Patrick Tolan et al. (2006, p. 569), le mécanisme causal entre pauvreté et violences est le stress : les difficultés économiques créeraient une forme d'anxiété propice à la violence. Un autre résultat de ces travaux est l'importance de la perception subjective du stress économique : les femmes dont le couple perçoit l'avenir de manière pessimiste sont plus à risques (Benson et al., 2004 ; Benson et Fox ; 2004).

Dans la sociologie américaine, la question de la race est toujours prégnante, dans la mesure où cette dernière a un effet important sur de nombreux outcomes sociaux et politiques (éducation, pauvreté, vote, etc.). Les corrélations entre race et criminalité sont particulièrement fortes (Unnever and Gabbidon, 2011). Les violences conjugales sont effectivement plus importantes dans la population noire (Sokoloff et Dupont, 2005). En particulier, les homicides conjugaux sont la première cause de mortalité pour les Africaines-Américaines entre 15 et 45 ans (West, 2004). Cependant, la corrélation entre race et violences conjugales disparaît quand on contrôle les facteurs socio-économiques (Rennison and Planty, 2003 ; Sokoloff et Dupont, 2005).

L'alcoolisme est fortement corrélé aux violences conjugales (Benson et al., 2003 ; Bair-Merritt et al., 2008 ; Gage, 2005 ; Kimuna et Djamba, 2008 ; Flake, 2005). Dans l'enquête ukrainienne, fondée sur l'analyse d'odds-ratios, l'alcool joue un rôle prépondérant dans l'étiologie des violences : les maris souvent ivres ont une probabilité 7 fois plus élevée de se montrer violents (Barrett et al., 2012). La plupart des revues de littérature mentionne le rôle de l'alcool, et en particulier R. Jewkes (2002). Dans un article plus ancien, Glenda Kaufman Kantor et M. A. Straus (1987) montraient que la consommation d'alcool n'est pas en soi une cause nécessaire ou suffisante pour expliquer les violences, mais que la conjonction de l'alcoolisme, de la pauvreté et d'attitudes qui légitiment la violence avait un fort pouvoir prédictif. La corrélation entre violences conjugales et alcool est cohérente compte tenu de la forte relation entre alcool et violences de toutes natures (Parker et Auerhahn, 1998).

Comment disent Natalie Sokoloff et Ida Dupont (2005, p. 44), « ces résultats remettent sérieusement en question l'affirmation des féministes mainstream selon laquelle la violence conjugale touche également toutes les femmes », laquelle «banalise à la fois les dimensions qui sous-tendent l'expérience des victimes de ces maltraitances particulières, et, plus important encore, la façon dont nous analysons la prévalence 
et l'impact de ces violences à l'encontre de ces victimes » (Kanuha, 1996, cité dans Sokoloff et Dupont, 2005, p. 41). Or, la violence touche également les couples homosexuels.

\section{Les violences dans les couples gays et lesbiens}

Les niveaux de violences sont les mêmes dans les couples hétérosexuels, gays (Merrill et Wolfe 2000) et lesbiens (Renzetti, 1992 ; Eaton et al., 2008), et les processus semblent similaires. Avec une définition large des violences (incluant les violences psychologiques et sexuelles), entre $25 \%$ et $50 \%$ des couples homosexuels sont concernés (McClennen, 2005 ; Murray and Mobley ; 2009). Les couples gays enregistreraient plus de violence physique, et la violence dans les couples lesbiens serait plus fréquente que dans les couples gays, mais moins sévère en termes de blessures physiques (Kulkin et al., 2007).

Un problème dans les études sur les couples gays et lesbiens est la taille des échantillons et la stratégie d'échantillonnage. La faible proportion des personnes homosexuelles dans la population ${ }^{9}$ et le biais de conformité relatif à la réprobation sociale de l'homosexualité limitent l'efficacité des enquêtes aléatoires à produire des gros échantillons. Cet obstacle est surmonté par Brian C. Kelly et. al (2011), qui ont constitué un échantillon de 1782 gays et 418 lesbiennes à New York et à Los Angeles. $16,6 \%$ s'identifient comme victime, $4,1 \%$ comme auteur, et $23,4 \%$ à la fois comme victime et auteur (sur les cinq dernières années). La bidirectionnalité de la violence suggère un pattern de violence situationnelle de couple. L'enquête ne relève pas de différence entre gays, lesbiennes et bisexuel-le-s dans la composition des violences (physiques ou psychologiques). Une autre étude systématique compare la prévalence des violences conjugales dans les couples homosexuels aux États-Unis et au Venezuela. Les taux de violence sont les mêmes, mais les enquêtés vénézuéliens ont une perception de la police et des tribunaux plus négative que les enquêtés américains (Burke et al., 2002).

Les processus qui causent ou font persister la violence dans les couples gays et lesbiens sont similaires aux dynamiques des couples hétérosexuels (Burke et Follingstad, 1999). La violence dans les couples gays et lesbiens est liée aux difficultés économiques (Kulkin et al., 2007), à la dépendance affective, à la jalousie (Eaton et al., 2008 ; McClennen, 2005), à l'alcool (Eaton et al., 2008 ; McClennen, 2005 ; Kelly et al., 2011). G. S. Merrill et V. A. Wolfe (2000, p. 1-2) notent au sujet de la violence dans les couples gays : "De même, les raisons les plus fréquemment citées pour rester - à savoir, l'espoir d'un changement et l'amour pour le conjoint semblent universelles à l'expérience d'être battu. »

Le contexte de l'homophobie entraîne des spécificités pour les hétéro- et homosexuels en rapport avec les violences. D'abord, la menace de l'outing (révéler l'homosexualité contre le gré du conjoint) est une source de conflit ou une raison pour la victime de rester dans une relation violente (Kulkin et al., 2007 ; Sokoloff et Dupont, 2005). Ensuite, la plupart des foyers et des associations sont spécialisés dans l'aide aux femmes hétérosexuelles (Kulkin et al., 2007 ; Eaton et al., 2008 ; Murray and

9. Dans la population française, $0,5 \%$ des femmes et $1,1 \%$ des hommes s'auto-identifient comme homosexuel-le-s, et $4 \%$ des femmes et $4,1 \%$ des hommes déclarent avoir eu des rapports homosexuels (Chauvin et Lerch, 2013). Pour étudier les violences dans les couples, il faut approcher la population restreinte. 
Mobley, 2009). Enfin, les gays et les lesbiennes tendent à percevoir la police et le système judiciaire comme des institutions homophobes (Kulkin et al., 2007). Le sida peut contraindre un homme malade à rester avec un conjoint violent (Kulkin et al., 2007 ; Merrill and Wolfe, 2000).

Si la violence conjugale est la violence des hommes contre les femmes, alors elle ne devrait pas exister au sein des couples homosexuels. Les violences dans les couples gays et lesbiens posent un problème aux théories selon lesquelles la violence conjugale est une violence des hommes contre les femmes, rendue possible par le patriarcat et/ou visant à la perpétuation du patriarcat (Burke et Follingstad, 1999; Eaton et al., 2008 ; McClennen, 2005). Comme l'écrivent Christine E. Murray et A. Keith Mobley (2009, p. 363-364) : «L'obstacle le plus fondamental pour les chercheurs sur les violences conjugales sur les couples de même sexe est que ces dernières confrontent le paradigme féministe dominant qui a lancé le mouvement contre les violences conjugales. L'essence et la philosophie du mouvement contre les violences conjugales (aussi appelé mouvement des femmes battues) est que le patriarcat et le sexisme sont les causes profondes des violences masculines contre les femmes dans les couples; donc, les femmes sont les victimes des violences conjugales d'auteurs mâles. » Pour Leslie K. Burke et Diane R. Follingstad (1999), l'un des obstacles aux progrès de la connaissance sur les violences dans les couples homosexuels est la « perception culturelle» que ces violences sont le fait des hommes sur les femmes.

\section{Les violences mesurées dans les enquêtes sont des conflits}

Récapitulons. Les enquêtes sur les déterminants montrent que les violences sont corrélées à la vulnérabilité sociale - pauvreté, chômage, alcoolisme. Autrement dit, les violences ne sont pas équitablement réparties parmi les milieux sociaux. Les enquêtes sur les couples gays et lesbiens montrent que les violences sont les mêmes dans les couples hétéro- et homosexuels. À tout le moins, cela suggère que les violences ne sont pas entièrement réductibles à la domination masculine. En revanche, l'idée que tous les couples se disputent, et que ces disputes prennent des formes plus violentes sous l'effet de la pauvreté et de l'alcool, est compatible avec le constat de la violence dans les couples homosexuels. Par conséquent, il est probable que l'essentiel des violences mesurées dans les enquêtes de victimation sont des violences de conflits ; ipso facto, il est probable que les analyses étiologiques des violences enquêtent sur les causes des disputes de couple (la violence situationnelle de couple). Ce raisonnement suggère que les violences de type «terrorisme intime » ne sont mesurées que de manière marginale par les enquêtes quantitatives.

La part prépondérante de la violence situationnelle de couple dans les violences conjugales est tellement évidente que certains auteurs ont fini par se distancier de la catégorie même de violence. Pour Janel M. Leone et al. (2004, p. 473), la typologie de M. P. Johnson est fondée sur le contrôle, pas sur la violence : le terrorisme intime implique le contrôle de la femme par le conjoint, tandis que la violence situationnelle de couple est une «simple» dispute. Le point empirique sur lequel se fondent J. M. Leone et al. (2004) est l'importance subjective des menaces pour les femmes, point qui rejoint les conclusions de D. M. Fergusson et al. (2005), pourtant de l'autre bord de la discussion. Comme il peut y avoir du contrôle sans violence, se focaliser sur la violence n'est pas nécessaire ; donc, la question de la violence est secondaire dans l'étude des violences conjugales. Ce que ne disent pas explicitement M. P. Johnson et ses coauteur-e-s, c'est que la violence est secondaire parce qu'il est 
désormais évident que la plupart de la violence conjugale n'est pas directement catégorisable comme violence sexiste. M. P. Johnson le dit presque : «De plus, c'est ce type [le terrorisme intime] que les gens ont à l'esprit quand ils entendent parler de violences conjugales. J'ai certainement conscience que, en tant que scientifiques, nous pouvons et nous devons opérationnaliser la violence conjugale avec précision (et ce même si nous ne pouvons le faire avec constance). Néanmoins, le terme "violences conjugales" charrie beaucoup de connotations qui peuvent ne pas correspondre à nos opérationnalisations. [...] Parfois j'aimerais qu'on puisse abandonner complètement le terme "violences conjugales". » (2005, p. 1127).

E. Stark radicalise cette position. Il part du principe que le champ des études sur la violence conjugale est dominé par une conception positiviste et behavioriste qui se focalise sur des incidents isolables de violence. Cette conception est celle du droit pénal, qui criminalise des actes isolables et hiérarchise leur gravité (Stark, 2006, p. $1019 ; 2010$, p. 203). Cette conception entraîne la confusion entre disputes (fights) et maltraitance unidirectionnelle (abuse). Ici E. Stark reprend à son compte la distinction de M. P. Johnson entre violence situationnelle de couple et terrorisme intime. Selon E. Stark (2006, p. 1024), les disputes ne sont pas un problème, parce que le recours à la violence est perçu comme légitime par les deux parties. Il distingue donc la violence «normative » (normative, qui sert à résoudre des disputes) de la violence «maltraitante» (abusive), et écrit : «Je reste agnostique sur la question de savoir si l'usage de la force est toujours inacceptable dans les relations ou les familles. » (2010, p. 202).

Le vrai problème, ce n'est donc pas la violence en soi, mais le fait de tenir son conjoint sous son emprise (ongoing entrapment) : c'est le « contrôle coercitif ». C'est un problème spécifiquement genré : les hommes ne se plaignent pas des violences de leur femme et ne cherchent pas à s'en protéger ${ }^{10}$. Le contrôle coercitif est le fait pour une femme de se retrouver dans une relation où elle est privée de son autonomie, de son estime de soi, et où elle se retrouve dans une situation d'esclavage domestique (domestic slavery) (Stark, 2006). E. Stark insiste sur le fait que la violence du contrôle coercitif n'est généralement pas très sérieuse, au sens où elle n'occasionne pas des blessures physiques graves : "Si la plupart des femmes battues subit [le contrôle coercitif] plutôt que la violence conjugale, cela expliquerait pourquoi la "maltraitance" continue après que les couples se séparent, pourquoi les violences "mineures" peuvent avoir des conséquences significatives, pourquoi les femmes battues sont piégées [entrapped] et développent un profil unique de problème, et pourquoi la définition en termes d'incidents isolables a échoué à susciter des interventions efficaces. » (Stark, 2006, p. 1021 ; voir aussi Stark, 2010).

Selon E. Stark, entre $60 \%$ et $80 \%$ des femmes qui cherchent de l'aide sont victimes de « contrôle coercitif ». Mais, pour lui, il ne fait pas de doute que « la majorité des actes violents identifiés par les enquêtes quantitatives tombe dans la catégorie des "disputes" et ne devraient pas être considérés comme de la "maltraitance" » (2010, p. 207). Il l'écrit plus clairement encore : "Dans la perspective du mouvement pour les femmes battues, la faiblesse de l'approche par la violence familiale réside moins dans ses méthodes ou ses résultats que dans son focus sur une réalité qui n'est que marginalement liée à notre but, qui est de mettre fin à l'emprise de la femme dans

10. Les taux d'arrestation (par la police) sont 7 fois plus élevés pour les hommes que pour les femmes (Tolan et al., 2006, p. 565) ; les hommes portent 5 fois moins plainte que les femmes et sont 5,4 fois plus poursuivis (Tjaden et Thoennes, 2000). 
sa vie personnelle. La question-clé n'est pas qui utilise la violence, mais quelles tactiques sont déployées pour opprimer les femmes dans leur vie personnelle. » (2006, p. 1020).

Concrètement, M. P. Johnson et E. Stark disent que la violence conjugale qui pose problème est la maltraitance unilatérale des hommes vers les femmes qui s'exprime dans des dynamiques de contrôle inscrites dans des rapports de genre. Quand la recherche mesure les actes de violence, elle enregistre les actes violents des disputes de couple, et non les actes violents des dynamiques de contrôle. Les recherches sur les déterminants montrent que les disputes dégénèrent dans un contexte de pauvreté et d'alcoolisme, et ont aussi bien lieu dans les couples hétéros que dans les couples gays et lesbiens.

Comprenons bien le problème : il y a un quasi-consensus pour dire que les enquêtes quantitatives enregistrent la violence des disputes, et que cette violence est qualitativement différente de la violence plus sournoise, plus ancrée dans la domination masculine, qui occasionne des traumatismes graves, qui est celle qui est problématisée socialement. Concrètement, ce raisonnement signifie que les enquêtes de victimation de type violence against women, qui n'interrogent pas les hommes et ne posent pas de questions d'autorévélation aux femmes, produisent des résultats douteux, parce que les faits enregistrés sont probablement des faits de disputes. Ces enquêtes choisissent de ne pas se donner les moyens de le savoir, en présupposant théoriquement la direction et la cause de la violence, tout en restant aveugles aux violences réellement genrées dont les femmes sont victimes, qui échappent largement aux enquêtes quantitatives.

Or, cette question n'est pas que théorico-méthodologique : le droit pénal reste calqué sur une définition de la violence conjugale comme actes violents isolables, et non comme dynamiques d'emprise et de contrôle telles que les conçoivent M. P. Johnson ou E. Stark. Cela implique que le système pénal punit indifféremment les auteurs de violences impliqués dans des disputes et les «terroristes intimes »; si E. Stark a raison sur la moindre gravité des violences dans les dynamiques de contrôle et d'emprise, le système pénal a même probablement tendance à punir moins sévèrement les terroristes intimes.

\section{Les conséquences de la criminalisation des violences conjugales aux États-Unis}

Aux États-Unis, depuis les années 1970, les violences conjugales sont passées de l'état d'affaires privées à celui de problème public. Les violences conjugales sont devenues un crime qui « offense les états forts et définis de la conscience collective » (Durkheim, 1893, p. 47), qui suscite une réprobation morale et requiert, par conséquent, une réponse pénale - le châtiment qui réaffirme l'attachement à la norme et, partant, la solidarité du groupe. Or, «le prix payé pour cette solidarité de sentiment est grand et parfois désastreux » (Mead, 1918, p. 592, voir également Garland, 1990). C'est l'argument que développe Kristin Bumiller à propos du système pénal américain. 


\section{Lutter contre les violences par le système pénal}

Les chercheur-e-s et militant-e-s inspirés par le Duluth Model ont appuyé le vote et la mise en œuvre de lois qui criminalisent les violences conjugales de la même façon que sont criminalisées les agressions dans l'espace public. Ils/elles voulaient que cesse l'indifférence de la police, que les violences conjugales soient traitées comme des crimes graves. Ils/elles partageaient l'optimisme d'alors envers l'efficacité du droit et la capacité de l'État à protéger les individus. Ils/elles ont initié et soutenu les formations pour sensibiliser les policiers aux violences conjugales, les poursuites pénales jusqu'au bout de la procédure (no-drop prosecution), le reporting obligatoire des faits à toutes les agences possiblement concernées, et les arrestations obligatoires (mandatory arrest, même en cas de retrait de plainte) (Bumiller, 2010). K. Bumiller s'intéresse à la façon dont « la relation entre un problème social [les violences conjugales] et les sciences sociales a évolué, et, dans ce cas, a formé une alliance avec un projet d'ingénierie sociale et de contrôle coercitif » (2010, p. 174).

Or, entre le début des années 1980 et les années 2000, le système pénal américain a connu une expansion historique : le taux d'incarcération est passé de 150 pour 100000 habitants à plus de 750 pour 100000 (plus de 7 fois les taux français ou allemands). La population carcérale américaine dépasse les 2,2 millions d'individus, et est si importante qu'elle diminue le taux de chômage (Western et Beckett, 1999). C'est un fait social majeur, qui affecte la stratification, l'organisation de l'Étatprovidence, et les lebenchancen de millions de personnes, et tout spécialement la minorité noire ${ }^{11}$. La criminalisation des violences conjugales s'inscrit dans ce contexte. Entre les années 1970 et les années 2000, le taux d'arrestation moyen après un appel d'urgence à la police pour violences conjugales est passé d'environ $10 \%$ à plus de $30 \%$ (Hirschel et al., 2007, p. 258). Dans les départements de police où sont appliquées les dispositions d'arrestation obligatoire, la probabilité d'arrestation est multipliée par deux par rapport aux départements où l'arrestation est discrétionnaire (Hirschel et al., 2007). Aujourd'hui, les violences commises dans les espaces privés sont plus susceptibles d'arrestations que les violences commises en public (Hirschel et al., 2007).

\section{Conséquences pour les femmes pauvres des minorités ethniques}

On a vu que la violence conjugale est concentrée dans les secteurs les plus vulnérables de la société. Aux États-Unis, cela concerne en premier lieu les minorités ethniques pauvres. La criminalisation et la répression des violences conjugales ont des conséquences négatives non seulement pour les hommes violents, mais aussi pour les femmes victimes. "Les femmes de couleur dans le mouvement anti-violence [conjugales] ont alerté contre l'idée d'investir trop lourdement dans les arrestations, l'emprisonnement et les poursuites comme réponses aux violences contre les femmes. [...] Et, pendant que le mouvement anti-violence travaille à améliorer les politiques d'arrestations, la sécurité quotidienne des populations de couleur est menacée par un policing plus agressif dont les conséquences sont un usage accru de la force, l'incarcération de masse et les violences policières. » (Richie, 2000, p. 1136).

11. En 2007, environ un tiers de tous les hommes noirs entre 20 et 30 ans sont soit en prison, soit en liberté surveillée (parole ou probation) (Tonry, 2009). Un jeune Africain-Américain qui sort du lycée sans qualification a $60 \%$ de risques d'aller en prison au moins une fois dans sa vie (Pettit et Western, 2004).

372, Revue française de sociologie, 56-2, 2015 
Le problème de la mise en œuvre des politiques de répression de la violence conjugale est que les agents qui s'en chargent sont des policiers, des juges, des gardiens de prison, des travailleurs sociaux - pas des militant-e-s contre la violence. Ces organisations mettent en œuvre des lois et des règlements. Quand elles se rendent compte que les femmes victimes de violences ont aussi des activités illégales (par exemple relevant de l'économie informelle), ou sont sans-papiers, ou ne s'occupent pas assez bien de leurs enfants, elles appliquent la loi et arrêtent également les femmes qui portaient plainte (Bumiller, 2010, p. 177). Les arrestations obligatoires produisent aussi un surcroît d'arrestations de femmes, notamment dans les cas de violences mutuelles, où les policiers arrêtent tout le monde. Dans certains états américains, cela peut concerner $30 \%$ du total des arrestations pour violences conjugales (Hirschel et al., 2007). La répression de la violence conjugale ne s'est donc pas seulement inscrite dans un mouvement de resocialisation des hommes, mais a accompagné une logique de maximisation des arrestations, des poursuites, et des incarcérations (Dixon, 2008, cité dans Bumiller, 2010). Pour K. Bumiller (2010, p. 183), «l'intervention de l'État [dans la sphère privée] pour arrêter les violences conjugales constitue une criminalisation sans précédent de la vie quotidienne », en particulier pour les femmes pauvres des ghettos noirs.

On a vu que les déterminants sociaux des violences maximisaient la probabilité que celles-ci surviennent dans les quartiers les plus pauvres. Les politiques répressives contre la violence conjugale entraînent donc une criminalisation accrue dans ces quartiers. On a aussi compris que, selon toute probabilité, une grande partie de ces violences ressortent de disputes de couple qui impliquent de la bidirectionnalité de la violence et ne sont pas forcément perçues par les participants comme de la maltraitance systématique (comme le «terrorisme intime » de M. P. Johnson ou le «contrôle coercitif » d'E. Stark). Les politiques répressives, et notamment celles qui obligent les policiers à arrêter toutes les personnes contre lesquelles une plainte a été déposée (même en cas de retrait de plainte), traitent tous les auteurs de violences sur leur femme comme des terroristes intimes, sans de donner les moyens de discerner les différents cas de figure.

Faire ces distinctions entre disputes et terrorisme intime (ou contrôle coercitif) permet de comprendre pourquoi les femmes restent avec des conjoints violents, ou pourquoi elles portent si peu plainte. Pour K. Bumiller (2010, p. 177), « [1]a question la plus compliquée et la plus inattendue à propos des politiques de mandatory enforcement $^{12}$ est la fréquence avec laquelle les victimes résistent ou se sentent attaquées par ces politiques ». On peut expliquer ce problème en invoquant la fausse conscience (aliénation, «aberration psychologique », «learned helplessness ») ; c'est d'ailleurs le sens des politiques de mandatory enforcement que de ne pas faire confiance à l'interprétation de la victime et de lui imposer le cadre d'analyse selon lequel elle est censée percevoir le tort qu'elle a subi. On peut aussi faire l'hypothèse que la violence incriminée est souvent réciproque, parce que survenant dans le cadre d'une dispute qui dégénère, et que cette violence qui n'est pas perçue par les victimes elles-mêmes comme du terrorisme intime se retrouve judiciairement étiquetée «violence conjugale », c'est-à-dire nécessairement illégitime ${ }^{13}$.

12. Le fait qu' une femme ne puisse retirer sa plainte, afin de forcer les poursuites judiciaires même en cas de réconciliation du couple.

13. M. P. Johnson et K. J. Ferraro (2000, p. 956) citent à ce sujet une étude selon laquelle après deux ans et demi, $25 \%$ des femmes qui étaient dans une relation violente sont restées dans cette relation violente, $43 \%$ sont parties et $32 \%$ ont négocié avec succès la fin de la violence. 


\section{Conséquences dans les débats féministes}

Cet état de fait a produit des débats dans les mouvements féministes américains. Pour Beth E. Richie (2000), le mouvement féministe dans les années 1970 a fait le choix stratégique de faire des violences le problème de toutes les femmes (sans distinction de statut social ou d'origine ethnique) pour problématiser d'une manière unifiée les questions de genre et ne pas contribuer à la stigmatisation des femmes pauvres et des minorités ethniques qui subissaient plus de violences. L'effet pervers de cette stratégie a été de que " "toutes les femmes" sont devenues une femme blanche de la classe moyenne » (Richie, 2000, p. 1135 ; voir aussi Sokoloff et Dupont, 2005). La femme blanche de la classe moyenne est devenue la «bonne victime » des violences conjugales.

Aux États-Unis, les femmes noires sont perçues comme plus irrationnelles, plus résistantes à la violence et plus agressives que les femmes blanches (Richie, 2000). Parce que la «bonne victime» de violence est perçue comme la victime d'une violence unilatérale de contrôle, la «bonne victime » ressemble plutôt à une femme blanche, tandis que les femmes noires voient les violences qu'elles subissent légitimées par leurs stéréotypes raciaux de comportement et souffrent d'un déficit de crédibilité comme victimes auprès des associations et surtout du système judiciaire ; les femmes noires passent donc pour de «mauvaises victimes » (West, 2004 ; Sokoloff et Dupont, 2005). La focalisation sur la dimension genrée de la domination a fonctionné aux dépens des dimensions économiques et raciales.

Du point de vue féministe noir, les féministes blanches ne sont pas touchées par les conséquences négatives de la criminalisation et de la répression de la violence conjugale. Elles ne voient donc pas le problème et continuent de travailler à une intensification de la répression avec un message inchangé sur le fait que «toutes les femmes sont à risque ». Pour B. E. Richie, cet état de fait est la raison pour laquelle les femmes de couleur sont déconnectées du mouvement féministe, puisque celui-ci contribue (indirectement, et sans le savoir) à leur oppression. L'article de B. E. Richie a eu un écho important, et de nombreuses publications depuis permettent de nuancer l'idée d'une recherche féministe aveugle aux effets négatifs ou contre-productifs de la criminalisation des violences conjugales dans le ghetto (Renzetti, 2001 ; Critical Resistance and Incite! 2003 ; DeKeseredy et Dragiewicz, 2007 ; Gondolf, 2007).

En somme, les réformes ont trop mis l'accent sur l'aspect répressif de la question ${ }^{14}$. Les travaux empiriques sur la question montrent que l'arrestation a un effet mineur et statistiquement non significatif sur la récidive des violences conjugales - au contraire du dépôt de plainte, très efficace (Felson et al., 2005). Les politiques d'arrestation obligatoire ne prennent pas en compte les conséquences néfastes pour les victimes elles-mêmes, notamment en termes économiques : « Ceux qui dépendent le plus des services publics sont les premiers à souffrir quand l'État-providence se tourne vers des formes de régulation et de contrôle étatique coercitif. » (Bumiller, 2010, p. 187). Dans la situation actuelle, le système pénal incarcère des individus impliqués dans des disputes de couple dont M. P. Johnson et E. Stark considèrent eux-mêmes que ce ne sont pas des «violences conjugales » au sens de maltraitance systématique.

14. Dans certains cas, les appels d'urgence à la police pour des faits de violences conjugales sont considérés comme des «nuisances»par les départements de police, lesquels peuvent menacer d'amendes les propriétaires de logements générateurs de «nuisances ». Cela aboutit à l'expulsion de la personne qui appelait la police pour se plaindre (Desmond et Valdez, 2012). 
Cette situation ne punit pas ceux qui devraient être punis, vulnérabilise des familles, coûte cher en incarcération, et dépense des ressources qui ne sont pas affectées aux politiques sociales contre la pauvreté ou l'alcoolisme, qui seraient plus efficaces pour réduire les violences.

\section{La construction des violences conjugales comme problème}

Ce travail fait le point sur l'état de la science sur les violences conjugales. La littérature scientifique distingue, d'une part, les disputes ordinaires et, d'autre part, la maltraitance systématique des femmes par les hommes (le terrorisme intime de M. P. Johnson, le contrôle coercitif d'E. Stark). Les disputes de couples sont fréquentes, concernent tous les types de couples (hétéros et gays) et peuvent dégénérer dans les contextes de pauvreté et d'alcoolisme en violences qui vont dans les deux sens, mais qui ont plus de chances de blesser les femmes, physiquement moins puissantes que les hommes. Ces données ne sont que partiellement cohérentes avec une théorie de la domination masculine. La maltraitance systématique est beaucoup moins fréquente, concerne essentiellement des victimes femmes et occasionne des traumatismes graves. Les données sur la maltraitance systématiques sont cohérentes avec une théorie de la domination masculine. Les enquêtes statistiques mesurent principalement les disputes de couple, et c'est pourquoi elles devraient poser des questions de victimation et d'autorévélation, et enquêter les femmes et les hommes, pour ne pas commettre l'erreur d'attribution qui consiste à dénoncer la maltraitance à partir de données sur les disputes de couples.

On le constate, les violences conjugales sont un objet saturé d'affects moraux. Par exemple, M. P. Johnson (1995, 2005) utilise le mot «terrorisme » pour désigner la maltraitance systématique qui ne ressort pas des disputes. Ce qui rend la sémantique de «terrorisme » légitime dans la sociologie des violences conjugales, c'est la réprobation dont ces violences font l'objet dans le champ scientifique. Cette réprobation a pu conduire Walter S. DeKeseredy et Molly Dragiewicz $(2007$, p. 882) à écrire une phrase qui explique, à mon avis, pourquoi les constats empiriques des enquêtes CTS sont ignorés : "Mettre fin à la maltraitance des femmes et aux autres formes de violence dans les relations intimes requiert aussi que les chercheurs dépassent le besoin d'essayer de "gagner un argument" au "nom de la science". » Cette réprobation peut être constituée en objet à analyser, en fait social pertinent.

S'intéresser à la réprobation morale de la violence conjugale permet de constater et d'expliquer la diffusion de normes juridiques et sociales concernant les violences contre les femmes. Cette démarche est originale parce qu'elle prend le contrepied des traditions positivistes et féministes. Les positivistes tendent à évacuer le sens pour ne s'occuper que du comptage des faits. Selon eux, seuls les faits (les cris, les gifles, les coups, etc.) sont objectivement mesurables, tandis que le sens est trop subjectif. Ils sont accusés par les féministes de ne pas s'intéresser au sens pour rester aveugles aux rapports de genre. Inversement, dans la littérature féministe, la question du sens ne se pose pas vraiment, puisque les violences sont conçues a priori comme de la domination masculine. Toute autre signification attribuée aux violences est fausse conscience. 
Le point de départ de la démarche qui s'intéresse à l'évolution de la réprobation des violences conjugales est le travail de Nitza Berkovitch et Karen Bradley (1999) sur la diffusion mondiale de normes pour l'éducation des femmes et contre l'excision. Elles montrent que pour recevoir l' «attention mondiale », les problèmes doivent être posés comme s'opposant aux «principes modernistes » : «l'universalisme, l'individualisme libéral et la rationalité ». Concevoir les violences conjugales comme un problème d'inégalité hommes-femmes, donc de "droits humains » (human rights, «droits de l'homme »), appartenant à la même catégorie que la prostitution forcée, le harcèlement sexuel ou l'excision - les violences contre les femmes - est plus efficace que de les concevoir comme un problème de redistribution économique ou de lutte contre l'alcoolisme.

Rachel S. Pierotti (2013) s'inspire de ce cadre d'analyse pour analyser la façon dont le problème de la violence conjugale est devenu formulé en termes de « violences contre les femmes ». À partir de la seconde moitié des années 1990, on observe dans le monde une rapide diffusion de normes juridiques et sociales concernant les violences contre les femmes. Cette diffusion culmine dans la campagne « Tous unis pour mettre fin à la violence à l'égard des femmes », lancée en 2008 par le secrétaire général de l'ONU Ban Ki-Moon, qui écrit sur son site internet: "Il y a une vérité universelle, applicable à tous les pays, toutes les cultures et toutes les populations : la violence contre les femmes n'est jamais acceptable, jamais excusable, jamais tolérable. » (cité dans Pierotti, 2013, p. 243).

$\mathrm{Au}$ terme d'une analyse sur 26 pays dont les gouvernements ont fait voter ou mis en œuvre des législations antiviolences conjugales, R. S. Pierotti (2013) montre que les évolutions démographiques, économiques, l'urbanisation, l'accès aux médias ou l'élévation du niveau d'éducation n'expliquent qu'une partie minoritaire de la tendance à la diffusion de normes contre les violences faites aux femmes. Elle s'appuie sur la théorie de la «société mondiale » (world society), qui étudie les relations entre les organisations internationales et les acteurs transnationaux comme les ONG et les associations professionnelles, pour en conclure à une explication par la diffusion mondiale de «scripts culturels ». Ces scripts culturels sont diffusés par les mouvements féministes occidentaux et déclinent les valeurs fondamentales de la world society : universalisme, individualisme libéral et égalité (Pierotti, 2013). Les travaux de N. Berkovitch et K. Bradley et de R. S. Pierotti montrent l'intérêt d'une analyse qui ne se limite pas à mesurer l'ampleur d'un phénomène réprouvé, mais qui cherche aussi à prendre pour objet la réprobation de ce phénomène.

François BonNeT

Pacte - CNRS BP 48

38040 Grenoble cedex

francois.bonnet@umrpacte.fr

376, Revue française de sociologie, 56-2, 2015 


\section{RÉFÉRENCES BIBLIOGRAPHIQUES}

ARChER J., 2000, «Sex Differences in Aggression Between Heterosexual Partners: A Meta-Analytic Review », Psychological Bulletin, 126, 5, p. 651-680.

BACHMAN R., 2000, « A Comparison of Annual Incidence Rates and Contextual Characteristics of Intimate-Partner Violence Against Women from the National Crime Victimization Survey (NCVs) and the National Violence Against Women Survey (Nvaws) », Violence Against Women, 6, 8, p. 839-867.

Bair-Merritt M. H., Holmes W. C., Holmes J. H., Feinstein J., Feudtner C., 2008, « Does Intimate Partner Violence Epidemiology Differ Between Homes With and Without Children? A Population-Based Study of Annual Prevalence and Associated Risk Factors », Journal of Family Violence, 23, 5, p. 325-332.

Barrett B. J., Habibov N., ChernyaK E., 2012, « Factors Affecting Prevalence and Extent of Intimate Partner Violence in Ukraine Evidence From a Nationally Representative Survey », Violence Against Women, 18, 10, p. 1147-1176.

Benson M. L., Fox G. L., 2004, Concentrated Disadvantage, Economic Distress, and Violence Against Women in Intimate Relationships, Washington (DC), National Institute of Justice.

Benson M. L., Fox G. L., Demaris A., Van Wyk J., 2003, « Neighborhood Disadvantage, Individual Economic Distress and Violence Against Women in Intimate Relationships », Journal of Quantitative Criminology, 19, 3, p. 207-235.

Berkovitch N., Bradley K., 1999, «The Globalization of Women's Status: Consensus/Dissensus in the World Polity », Sociological Perspectives, 42, 3, p. 481-498.

BourgoIs P., 2010, «Recognizing Invisible Violence: A Thirty-Year Ethnographic Retrospective » dans B. RYLKO-BAUER, L. WhITEFORD, P. FARMER (eds.), Global Health in Times of Violence, Santa Fe (NM), School for Advanced Research Press, p. $17-40$.

Bumiller K., 2010, «The Nexus of Domestic Violence Reform and Social Science: From Instrument of Social Change to Institutionalized Surveillance », Annual Review of Law and Social Science, 6, p. 173-193.

Bureau of Justice Statistics, 2005, Family Violence Statistics. Including Statistics on Strangers and Acquaintances, Washington (DC), Us Department of Justice: http://www.bjs.gov/content/pub/pdf/fvs03.pdf [consulté le 28-04-2015].

BurKe L. K., Follingstad D. R., 1999, « Violence in Lesbian and Gay Relationships: Theory, Prevalence, and Correlational Factors », Clinical Psychology Review, 19, 5, p. 487-512.

Burke T. W., Jordan M. L., Owen S. S., 2002, «A Cross-National Comparison of Gay and Lesbian Domestic Violence », Journal of Contemporary Criminal Justice, 18,3 , p. 231-257.

Chauvin S., Lerch A., 2013, Sociologie de l'homosexualité, Paris, La Découverte (Repères, 618).

Critical Resistance And Incite! 2003, «Critical Resistance-Incite! Statement on Gender Violence and the Prison-Industrial Complex », Social Justice, 30, 3, p. 141-150. 
DeKeseredy W. S., Dragiewicz M, 2007, « Understanding the Complexities of Feminist Perspectives on Woman Abuse: A Commentary on Donald G. Dutton's Rethinking Domestic Violence », Violence Against Women, 13, 8, p. 874-884.

Desmond M., VAldez N., 2013, « Unpolicing the Urban Poor Consequences of ThirdParty Policing for Inner-City Women», American Sociological Review, 78, 1, p. 117-141.

Dixon J., 2008, « Mandatory Domestic Violence Arrest and Prosecution Policies: Recidivism and Social Governance », Criminology and Public Policy, 7, 4, p. 663-670.

Dobash R. E., Dobash R. P., 1979, Violence Against Wives: A Case against the Patriarchy, London, Open Books.

Durkheim É., [1893] 1998, De la division du travail social, Paris, Presses universitaires de France (Quadrige).

Eaton L., Kaufman M., Fuhrel A., Cain D., Cherry C., Pope H., Kalichman S. C., 2008, «Examining Factors Co-Existing With Interpersonal Violence in Lesbian Relationships », Journal of Family Violence, 23, p. 697-705.

EIsikovits Z., Winstok Z., Fishman G., 2004, « The First Israeli National Survey on Domestic Violence », Violence Against Women, 10, 7, p. 729-748.

FELson M., 1994, Crime and Everyday Life. Insight and Implications for Society, Thousands Oaks (CA), Pine Forge Press.

Felson R. B., Ackerman J. M., Gallagher C. A., 2005, « Police Intervention and the Repeat of Domestic Assault », Criminology, 43, 3, p. 563-588.

Felson R. B., Ackerman J., Yeon S. J., 2003, «The Infrequency of Family Violence », Journal of Marriage and Family, 65, 3, p. 622-634.

Fergusson D. M., Horwood L. J., Ridder E. M., 2005, «Partner Violence and Mental Health Outcomes in a New Zealand Birth Cohort », Journal of Marriage and Family, 67, 5, p. 1103-1119.

FIEBERT M. S., 1997, « References Examining Assaults by Women on their Spouses or Males Partners: An Annotated Bibliography », Sexuality and Culture, 1, p. 273-286.

FlaKe D. F., 2005, «Individual, Family, and Community Risk Markers for Domestic Violence in Peru », Violence Against Women, 11, 3, p. 353-373.

GaGe A. G., 2005, «Women's Experience of Intimate Partner Violence in Haiti », Social Science \& Medicine, 61, 2, p. 343-364.

Garland D., 1990, Punishment and Modern Society. A Study in Social Theory, Chicago (IL) The University of Chicago Press (Studies in Crime and Justice).

Gelles R. J., 1985, « Family Violence », Annual Review of Sociology, 11, p. 347-367.

Gondolf E. W., 2007, « Theoretical and Research Support for the Duluth Model: A Reply to Dutton and Corvo », Aggression and Violent Behavior, 12, 6, p. 644-657.

Graham-Kevan N., Archer J., 2003, «Intimate Terrorism and Common Couple Violence. A Test of Johnson's Predictions in Four British Samples », Journal of Interpersonal Violence, 18, 11, p. 1247-1270.

HaJ-Yahia M. M., 2000, «The Incidence of Wife Abuse and Battering and Some Socio-Demographic Correlates as Revealed by Two National Surveys in Palestinian Society », Journal of Family Violence, 15, 4, p. 347-374. 
Hirschel D., Buzawa E., Pattavina A., Faggiani D., 2007, « Domestic Violence and Mandatory Arrest Laws: To What Extent do they Influence Police Arrest Decisions? », The Journal of Criminal Law and Criminology, 98, 1, p. 255-298.

Jewkes R., 2002, «Intimate Partner Violence: Causes and Prevention », The Lancet, 359,9315 , p. $1423-1429$.

Johnson M. P., 1995, « Patriarchal Terrorism and Common Couple Violence: Two Forms of Violence Against Women », Journal of Marriage and the Family, 57, 2, p. 283-294.

Johnson M. P., 2005, « Domestic Violence: It's Not About Gender - Or Is It?», Journal of Marriage and Family, 67, 5, p. 1126-1130.

Johnson M. P., 2006, « Conflict and Control. Gender Symmetry and Asymmetry in Domestic Violence », Violence Against Women, 12, 11, p. 1003-1018.

JoHnson M. P., 2010a, « Langhinrichsen-Rolling's Confirmation of the Feminist Analysis of Intimate Partner Violence: Comment on "Controversies Involving Gender and Intimate Partner Violence in the United States" », Sex Roles, 62, 3-4, p. 212-219.

Johnson M. P., 2010b, A Typology of Domestic Violence: Intimate Terrorism, Violent Resistance, and Situational Couple Violence, Lebanon (NH), University Press of New England.

Johnson M. P., Ferraro K. J., 2000, « Research on Domestic Violence in the 1990s: Making Distinctions », Journal of Marriage and Family, 62, 4, p. 948-963.

JoRdAn C. E., CAMPBell R., Follingstad D., 2010, « Violence and Women's Mental Health: The Impact of Physical, Sexual, and Psychological Aggression », Annual Review of Clinical Psychology, 6, 1, p. 607-628.

Kantor G. K., Straus M. A., 1987, «The "Drunken Bum" Theory of Wife Beating », Social Problems, 34, 3, p. 213-230.

Kelly B. C., Izienicki H., Bimbi D. S., Parsons J. T., 2011, « The Intersection of Mutual Partner Violence and Substance Use Among Urban Gays, Lesbians, and Bisexuals », Deviant Behavior, 32, 5, p. 379-404.

KImmel M. S., 2002, "Gender Symmetry" in Domestic Violence: A Substantive and Methodological Research Review», Violence Against Women, 8, 11, p. $1332-1363$.

Kimuna S. R., Duamba Y. K., 2008, « Gender Based Violence: Correlates of Physical and Sexual Wife Abuse in Kenya », Journal of Family Violence, 23, 5, p. 333-342.

Kulkin H. S., Williams J., Borne H. F., DE la Bretonne D., Laurendine J., 2007, « A Review of Research on Violence in Same-Gender Couples », Journal of Homosexuality, 53, 4, p. 71-87.

Kury H., Obergfell-Fuchs J., Woessner G., 2004, « The Extent of Family Violence in Europe. A Comparison of National Surveys », Violence Against Women, 10, 7, p. $749-769$.

LANGHINRICHSEN-Rohling J., 2010, «Controversies Involving Gender and Intimate Partner Violence in the United States », Sex Roles, 62, 3-4, p. 179-193.

Leone J. M., Johnson M. P., Cohan C. L., Lloyd S. E., 2004, « Consequences of Male Partner Violence for Low? Income Minority Women », Journal of Marriage and Family, 66, 2, p. 472-490. 
McClennen J. C., 2005, «Domestic Violence Between Same-Gender Partners: Recent Findings and Future Research », Journal of Interpersonal Violence, 20, 2, p. 149-154.

Mead G. H., 1918, « The Psychology of Punitive Justice », American Journal of Sociology, 23, 5, p. 577-602.

Medina-Ariza J., BARberet R., 2003, « Intimate Partner Violence in Spain Findings From a National Survey », Violence Against Women, 9, 3, p. 302-322.

Merrill G. S., Wolfe V. A., 2000, «Battered Gay Men: An Exploration of Abuse, Help Seeking, and Why they Stay », Journal of Homosexuality, 39, 2, p. 1-30.

Murray C. E., Mobley A. K., 2009, « Empirical Research About Same-Sex Intimate Partner Violence: A Methodological Review », Journal of Homosexuality, 56, 3, p. 361-386.

Murray C. E., Mobley A. K., Buford A. P., Seaman-Dejohn M. M., 2007, «Same-Sex Intimate Partner Violence: Dynamics, Social Context, and Counseling Implications », Journal of LGBT Issues in Counseling, 1, 4, p. 7-30.

Parker R. N., Auerhahn K., 1998, « Alcohol, Drugs, and Violence », Annual Review of Sociology, 24, p. 291-311.

Pettit B., Western B., 2004, « Mass Imprisonment and the Life Course: Race and Class Inequality in US Incarceration », American Sociological Review, 69, 2, p. 151-169.

PIEROTTI R. S., 2013, « Increasing Rejection of Intimate Partner Violence. Evidence of Global Cultural Diffusion », American Sociological Review, 78, 2, p. 240-265.

Rennison C., Planty M., 2003, « Nonlethal Intimate Partner Violence: Examining Race, Gender, and Income Patterns », Violence and Victims, 18, 4, p. 433-443.

Renzetti C. M., 1992, Violent Betrayal: Partner Abuse in Lesbian Relationships, Newbury Park (CA), Sage.

RenZetTI C. M., 2001, «"One Strike and You're Out”. Implications of a Federal Crime Control Policy for Battered Women », Violence Against Women, 7, 6, p. 685-698.

RichiE B. E., 2000, «A Black Feminist Reflection on the Antiviolence Movement », Signs, 25, 4, p. 1133-1137.

SAunders D. G., 2002, «Are Physical Assaults by Wives and Girlfriends a Major Social Problem? A Review of the Literature », Violence Against Women, 8, 12, p. 1424-1448.

Saunders D. G., Browne A., 2000, «Intimate Partner Homicide » dans R. T. Ammerman, M. Hersen (eds.), Case Studies in Family Violence, New York (NY), Plenum, p. 415-449 [2 $2^{\mathrm{e}}$ ed.].

SCHEPER-Hughes N., Bourgois P., 2004, «Introduction: Making Sense of Violence » dans N. SchePer-Hughes, P. Bourgois (eds.), Violence in War and Peace. An Anthology, Oxford, Blackwell, p. 1-32.

Simon T. R., Anderson M., Thompson M. P., Crosby A. E., Shelley G., Sacks J. J., 2001, «Attitudinal Acceptance of Intimate Partner Violence Among us Adults », Violence and Victims, 16, 2, p. 115-126.

Sokoloff N. J., Dupont I., 2005, « Domestic Violence at the Intersections of Race, Class, and Gender: Challenges and Contributions to Understanding Violence Against Marginalized Women in Diverse Communities », Violence Against Women, 11, 1, p. 38-64. 
STARK E., 2006, « Commentary on Johnson's "Conflict and Control: Gender Symmetry and Asymmetry in Domestic Violence" », Violence Against Women, 12, 11, p. 1019-1025.

Stark E., 2010, «Do Violent Acts Equal Abuse? Resolving the Gender Parity/ Asymmetry Dilemma », Sex Roles, 62, 3-4, p. 201-211.

Steinmetz S. K., 1977, «The Battered Husband Syndrome », Victimology, 2, 3-4, p. 499-509.

Stinchcombe A. L., 1963, «Institutions of Privacy in the Determination of Police Administrative Practice », American Journal of Sociology, 69, 2, p. 150-160.

Straus M. A., 1979, « Measuring Intrafamily Conflict and Violence: The Conflict Tactics (СТ) Scales », Journal of Marriage and the Family, 41, 1, p. 75-88.

Straus M. A., 2006, «Future Research on Gender Symmetry in Physical Assaults on Partners », Violence Against Women, 12, 11, p. 1086-1097.

Straus M. A., Gelles R. J., Steinmetz S. K., 1980, Behind Closed Doors: Violence in the American Family, Garden City (NY), Anchor/Doubleday.

Straus M. A., Hamby S. L., Boney-McCoy S., Sugarman D. B., 1996, « The Revised Conflict Tactics Scales (CTS2) Development and Preliminary Psychometric Data », Journal of Family Issues, 17, 3, p. 283-316.

TJaden P., Thoennes N., 1999, Extent, Nature, and Consequences of Intimate Partner Violence: Findings from the National Violence Against Women Survey, Washington (DC), National Institute of Justice/Centers for Disease Control and Prevention.

TJaden P., Thoennes N., 2000, «Prevalence and Consequences of Male-to-Female and Female-to-Male Intimate Partner Violence as Measured by the National Violence Against Women Survey », Violence Against Women, 6, 2, p. 141-161.

Tolan P., Gorman-Smith D., Henry D., 2006, « Family Violence », Annual Review of Psychology, 57, p. 557-583.

Tonry M., 2009, «Explanations of American Punishment Policies: A National History », Punishment \& Society, 11, 3, p. 377-394.

United Nations Population Fund, 2000, The State of World Population: Lives together, Worlds Apart-Men and Women in a Time of Change, New York (NY), United Nations.

Unnever J. D., Gabbidon S. L., 2011, Race, Racism, and Crime: A Theory of African American Offending, London, Routledge.

West C. M., 2004, « Black Women and Intimate Partner Violence New Directions for Research », Journal of Interpersonal Violence, 19, 12, p. 1487-1493.

Western B., BecketT K., 1999, «How Unregulated is the us Labor Market? The Penal System as a Labor Market Institution », American Journal of Sociology, 104, 4, p. 1030-1060. 


\section{AbSTRACT \\ Intimate partner violence, gender and criminalization: a synthesis of American debates}

The article reviews American literature on domestic or intimate partner violence (IPV). Several major surveys have been conducted in the us by researchers with different theoretical positions (e.g., family violence versus violence against women); the survey findings have elicited much debate. This review of the literature offers a detailed summary of those debates-on gender symmetry, whether violence is bidirectional, how to define IPV, its etiology, legal responses to it-with the aim of helping to construct the research object called "intimate partner violence." The disapproval and condemnation elicited by such violence is also an object of analysis.

Key words. INTIMATE PARTNER VIOLENCE - VIOLENCE AGAINST WOMEN - GENDER - CRIMINALIZATION - UNITED STATES

\section{ZUSAMMENFASSUNG}

\section{Häusliche Gewalt, Geschlecht und Kriminalisierung: die Synthese der amerikan- ischen Debatte}

Diese Arbeit beschäftigt sich mit der amerikanischen Literatur zur Gewalt in Paarbeziehungen. Es wurden mehrere großen Untersuchungen geführt durch Forscher und Forcherinnen mit unterschiedlichen theoretischen Orientierungen (Familiengewalt versus Gewalt gegen Frauen). Die Ergebnisse dieser Untersuchungen führten zu Debatten. Diese Literaturübersicht möchte die Debatten wiedergeben — zur Geschlechtersymmetrie und zur Bidirektionalität der Gewalttaten, zu deren Definition, Ätiologie und Strafrechtlichen Behandlung, - um zur Konstruktion des Objekts "Gewalt in Paarbeziehungen" beizutragen, da die Mißbilligung, die die Gewalt in Paarbeziehungen hervorruft selbst ein Untersuchungsobjekt darstellt.

Wörter Schlüssel. Gewalt in PaARBeziehungen - Gewalt gegen Frauen Geschlecht - Kriminalisierung - Vereinigte StaAten

\section{RESUMEN}

Violencias conyugales, género y criminalización: síntesis de los débates en América del Norte

Este trabajo trata de la literatura norteamericana sobre las violencias conyugales. Cien-
tíficos con presupuestos teóricos diferentes (violencias familiares versus violencia contra
las mujeres) llevaron a cabo diversas investigaciones de gran escala cuyos resultados
han generado debates. El examen de esta literatura pretende restituir estos debates
- sobre la simetría de género y la bidirectionnalidad de las violencias, su definición, su 
etiología, su tratamiento penal - con el fin de contribuir a la construcción del objeto "violencias conyugales", la reprobación que suscitan estas violencias constituyendo un objeto de estudio en si.

Palabras claves. VIOLENCIA CONYUGAL - VIOLENCIA CONTRA LAS MUJERES - GÉNERO CRIMINALIZACIÓN - Estados Unidos de AmÉRICA 